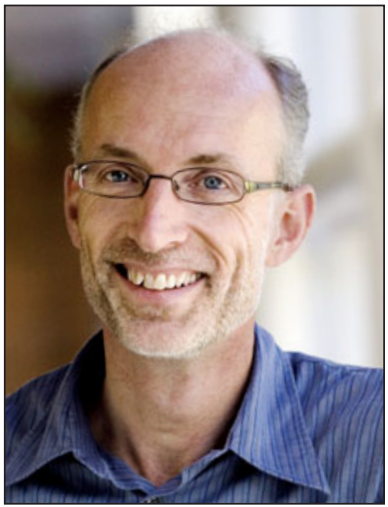

Stuart S.P. Parkin to receive 2012 Von Hippel Award for spintronics

$\mathbf{T}_{\mathrm{t}}$ he 2012 Von Hippel Award, the Materials Research Society's highest honor, will be presented to Stuart S.P. Parkin, IBM Fellow, Manager of the Magnetoelectronics Group, and Director of the IBM-Stanford Spintronic Science and Applications Center (SpinAps) at IBM Almaden Research Center in San Jose, Calif. Parkin is being recognized for "pioneering contributions to the science and technology of spintronic materials, particularly in establishing the fundamental foundations of spin-engineered magnetic heterostructures and demonstrating artificial atomically-layered magnetic multilayers for applications in field sensing, magnetic memory and data storage devices." Parkin will accept the honor during the awards ceremony at the 2012 MRS Fall Meeting in Boston.

Parkin, who received the inaugural MRS Outstanding Young Investigator Award in 1991 and joined the MRS inaugural class of fellows in 2008, essentially created and defined the research field of spintronics. After the initial discovery of the giant magnetoresistance (GMR) effect in 1998 by Nobel laureates Peter Grunberg (KFA, Jülich) and Albert Fert (Université Paris-Sud), Parkin opened the door for applications of GMR. One of his remarkable contributions to fundamental science was his discovery of the oscillatory interlayer exchange coupling and related GMR effect in metallic superstructures. Parkin demonstrated that oscillatory interlayer coupling is a universal property of nearly all transition and noble-metal-based multilayers, thus developing a method of designing specific materials with special proper- ties-here the GMR effect-based on their superstructure. His systematic investigation of a large number of elemental combinations enabled Parkin to find the unique combination of ferromagnetic and nonmagnetic metals that gives rise to a very large magnetoresistance effect at room temperature. These designed "nanoengineered devices" with sophisticated properties, together with the development of a rapid and versatile sputtering method for thin-film structure exploration rendered the application of the GMR effect possible.

Parkin and his colleagues furthermore developed novel magnetic-field sensing devices, which they named spin valves, for use in magnetic disk-drive read heads. Structures based on Parkin's discoveries and inventions were used in IBM's first GMR recording head in 1997, and soon thereafter in all manufacturers' hard-disk drives as well as in many other magnetic-field sensors, magnetic memory elements, and antiferromagnetically coupled magnetic media for high-density harddisk drives. Spin valves are also now used for sensors in the automotive industry and for biomedical applications.

The GMR-based spin-valve field sensor was superceded by the giant tunnel magnetoresistance effect (TMR), another of Parkin's discoveries. Parkin demonstrated that the TMR effect, found when electrons tunnel between ferromagnetic layers separated by an ultrathin tunnel barrier, could be dramatically enhanced by replacing conventional tunnel barriers of amorphous aluminum oxide by crystalline layers of $\mathrm{MgO}$. The resistance of the device depends on the magnetic orientation of the layers' moments, just as for GMR, but the physics of TMR is distinct from that of GMR. The TMR effect is enhanced due to resonant spindependent tunneling through the $\mathrm{MgO}$ barrier, which acts as a spin filter.

The race track memory, one of Parkin's current projects, is a radically new storage-memory technology using spin currents to directly manipulate the magnetic state of nanoscale magnetic regions - magnetic domain walls - within magnetic nanowires. This concept is based on the storage of information in three-dimensional arrays of magnetic nanowires and using current-induced displacement of the domain walls along the nanowires. This device aims to match the massive storage capacity of hard disks but with the advantage of a purely electronic operation. A two-dimensional version of the device could also compete with flash memory. The race track memory concept was selected as one of the MIT Technology Review's 2009 list of "10 emerging technologies that can change the way we live."

Parkin received his $\mathrm{BA}$ and $\mathrm{PhD}$ degrees from the University of Cambridge and joined IBM as a postdoctoral fellow in 1982, becoming a permanent member of the staff the following year. In 1999, he was named an IBM Fellow, IBM's highest technical honor. Parkin is also a Fellow of the Royal Society (elected 2000), the American Physical Society, the Institute of Physics (London), the Institute of Electrical and Electronics Engineers, the American Association for the Advancement of Science, and the American Academy of Arts and Sciences (elected 2009). He is also a Member of the National Academy of Science (elected 2008) and the National Academy of Engineering (elected 2009). In 2012, Parkin was elected an Honorary Fellow of the Indian Academy of Sciences. His honors include a Humboldt Research Award (2004), the 1999-2000 American Institute of Physics Prize for Industrial Applications of Physics, the European Physical Society's Hewlett-Packard Europhysics Prize (1997), the American Physical Society's International New Materials Prize (1994), the IUPAP Magnetism Prize and Neel Medal (2009), and the Charles Vernon 
Boys Prize from the Institute of Physics, London (1991). In 2001, he was named $R \& D$ Magazine's first Innovator of the Year. Parkin has authored $\sim 00$ papers and has $\sim 93$ issued patents.

The MRS Von Hippel Award includes a $\$ 10,000$ cash prize, honorary membership in MRS, and a unique trophy - a mounted ruby laser crystal, symbolizing the many faceted nature of materials research. The award recognizes those qualities most prized by materials sci- entists and engineers-brilliance and originality of intellect, combined with vision that transcends the boundaries of conventional disciplines, as exemplified by the life of Arthur von Hippel (http:// vonhippel.mrs.org).

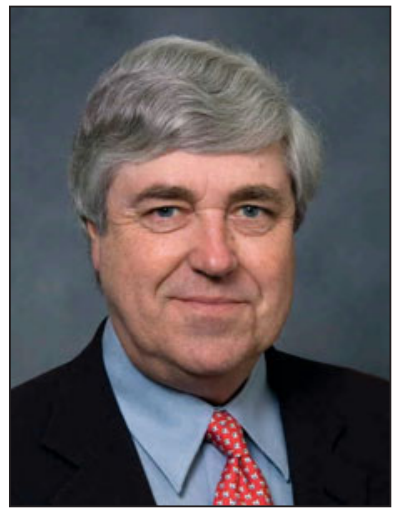

\section{Robert Sinclair} selected for 2012 David Turnbull Lectureship

$\mathbf{T}$ he Materials Research Society's David Turnbull Lectureship recognizes the career of a scientist who has made outstanding contributions to understanding materials phenomena and properties through research, writing, and lecturing, as exemplified by the late David Turnbull of Harvard University. This year Robert Sinclair, chair of the Materials Science and Engineering Department of Stanford University and Charles M. Pigott Professor in the School of Engineering, and founding director of the Stanford Nanocharacterization Laboratory, has been selected to give the 2012 Turnbull Lecture. Sinclair is cited for his "original contributions to the understanding of atomic arrangements in solids and their relationship to diverse materials phenomena including martensitic transformations, dislocation interactions with interfaces, phase equilibria in complex thin film systems, and nanoscale interactions in soft matter, for seminal contributions to in-situ and high resolution transmission electron microscopy, development of their combined use, and for passionate and dedicated teaching, advising, and academic leadership." Sinclair will be presented with the award at the 2012 MRS Fall Meeting in Boston.

Broadly acknowledged as a pioneering contributor to the evolution of ana- lytical and high-resolution electron microscopy as an essential research tool for developing fundamental understanding of the relationship between the atomic structure of materials, defects, phase transformations and equilibria, and diverse properties of interest, Sinclair's work has encompassed a broad range of materials including semiconductor materials ( $\mathrm{Si}$ and III-V) and metal silicides, magnetic recording media, shapememory alloys, hard materials, and most recently soft (nanobio) matter. He developed techniques for in situ transmission electron microscopy (TEM) observations of transient phenomena at the atomic level, thereby producing the first direct observations of atomic behavior during controlled materials reactions.

Under his leadership, electron microscopy in particular and materials research more broadly has, at Stanford University, experienced a dramatic growth in capabilities, as most recently signified by the addition of the Nanocharacterization Laboratory. Sinclair has also taken passionate interest in developing introductory and graduate level courses in materials science and characterization that have trained hundreds of students, many of whom have chosen to specialize in materials research. His transition to the Department chair position has resulted in substantial improvements in the undergraduate and graduate curriculum in the Department, and the addition of young faculty, world renowned for their research in diverse fields such as energy and environmental materials, plasmonics, and nanophotonics.

In addition to teaching at Stanford, Sinclair has long been active in the education of researchers outside of his own university. He was among the first faculty members to travel to China in 1980, where he taught a course on TEM theory and practice. He has presented extensively in conferences worldwide and is known for his lucid delivery, often interspersed with levity, and for making difficult concepts simple to grasp. He has also taught extensively in industrial settings in order to familiarize workers with the efficacy and flexibility of various analytical techniques. Simultaneously, he has always encouraged independent thinking, allowing his students to flourish on their own, and to develop their own research areas instead of following his model.

Sinclair received his BA (1968) and $\mathrm{PhD}$ (1972) degrees in materials science from Cambridge University. He has over 200 refereed publications and three patents. His honors include the Robert Lansing Hardy Gold Medal, The Metallurgical Society of AIME; Eli Franklin Burton Award, Electron Microscopy Society of America; Alfred P. Sloan Foundation Fellowship; Marcus E. Grossman Award, American Society for Metals; Excellence in Undergraduate Teaching twice, once from the Stanford University Society for Black Scientists and Engineers and again from the Stanford Society of Chicano/Latino Engineers and Scientists; and Fellow (inaugural class) as well as Distinguished Scientist, Physical Sciences, of the Microscopy Society of America. 